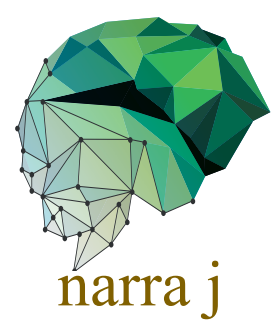

Original Article

\title{
Disruption of childhood vaccination during the COVID-19 pandemic in Indonesia
}

\author{
Marhami Fahriani', Samsul Anwar², Amanda Yufika3, Bakhtiar Bakhtiar4, Elly \\ Wardani5, Wira Winardi6, Kaitlyn B. Akel7, Abram L. Wagner7 and Harapan \\ Harapan 1,8,9*
}

${ }^{1}$ Medical Research Unit, School of Medicine, Universitas Syiah Kuala, Banda Aceh, Indonesia; ${ }^{2}$ Department of Statistics, Faculty of Mathematics and Natural Sciences, Universitas Syiah Kuala, Banda Aceh, Indonesia; 3Department of Family Medicine, School of Medicine, Universitas Syiah Kuala, Banda Aceh, Indonesia; 4Department of Pediatric, School of Medicine, Universitas Syiah Kuala, Banda Aceh, Indonesia; 5Department of Nursing Leadership and Management, Faculty of Nursing, Universitas Syiah Kuala, Banda Aceh, Indonesia; ${ }^{6}$ Department of Pulmonology and Respiratory Medicine, School of Medicine, Universitas Syiah Kuala, Banda Aceh, Indonesia; 7Department of Epidemiology, University of Michigan, Ann Arbor, Michigan, United States of America; ${ }^{8}$ Tropical Disease Centre, School of Medicine, Universitas Syiah Kuala, Banda Aceh, Indonesia; ${ }^{9}$ Department of Microbiology, School of Medicine, Universitas Syiah Kuala, Banda Aceh, Indonesia

*Corresponding author: harapan@unsyiah.ac.id

\section{Abstract}

The aim of this study was to assess the magnitude of childhood vaccination disruption and to determine the predictors of delaying childhood vaccinations during the coronavirus disease 2019 (COVID-19) pandemic among Indonesian parents. We conducted a nationwide, online, cross-sectional study. A set of questionnaires assessed the disruption of childhood vaccinations and possible explanatory variables, including demographic characteristics, current underlying disease, exposure to and confidence in COVID-19 information, perceived risk, attitude and practice on vaccination, and COVID-19 prevention practice. A multivariable linear regression was used to characterize the relationship between explanatory variables and delayed childhood vaccination. We included 1137 respondents for analysis, of which $52.6 \%$ were males and $58.4 \%$ participants aged between 31 and 50-years old. Disruption of childhood vaccination service in local health facilities was reported in $42.2 \%$ (480/1137) of respondents and 13.3\% (193/1137) of respondents explained that their children could not be vaccinated because a healthcare facility temporary stopped the vaccination service. Of all respondents, 312 (27.4\%) delayed vaccinating their children for a compulsory vaccination shot. Factors associated with higher odds of delaying compulsory vaccinations for children were employment and chronic disease status, flu vaccination status in the past year, and prior COVID-19 infection. In conclusion, there was a significant disruption of childhood vaccination practices in Indonesia during the COVID-19 pandemic. This is not only due to healthcare closures but also due to parents' practice - delaying the compulsory childhood vaccination for their children. We urge the government to strengthen strategies to ensure childhood vaccination services are available to all children in the country during a pandemic.

Keywords: COVID-19, SARS-CoV-2, vaccination, childhood vaccination

\section{Introduction}

Childhood vaccination programs have rapidly expanded worldwide after the establishment of the Expanded Program on Immunization by the World Health Organization (WHO) in 1974 [1]. In 2020, the WHO launched the Immunization Agenda 2030, which aims to reduce the mortality 
and morbidity from vaccine-preventable disease during the life course by increasing equitable access and strengthening immunization programs within primary health care across the world [1]. Within Indonesia, specifically, there has been a steady increase in vaccination coverage over the past three decades [2]. However, since the WHO declared the coronavirus disease 2019 (COVID-19) a global pandemic on March 11, 2020, several countries reported a decline in vaccination coverage [3-5]. A decline of $19.8 \%$ in measles-mumps-rubella (MMR) vaccination was reported in the UK compared to the same period in 2019 [6]. WHO also warned of a significant decrease in immunization of three-dose diphtheria-tetanus-pertussis vaccine (DTP3) for the first 4 months of 2020 [7]. Saudi Arabia also reported a decline in vaccination visits from March to May 2020 compared to the same period in 2017-2019 [8].

The COVID-19 pandemic raised multiple concerns around the globe, relating to public health and socioeconomic and political systems [9]. With stay-at-home orders, physical distancing, domestic and international travel restrictions in place to mitigate viral transmission, concern for exposing children to COVID-19 while getting immunizations in medical centers was expected [10, 11]. However, in some places, the decline in routine vaccines has been staggering. One study in Sindh province, Pakistan, showed a severe drop in routine immunizations for children during the pandemic, with $50 \%$ of children missing essential vaccines across the province, with higher proportions missed in rural areas [12]. Moreover, a risk-benefit analysis in Africa showed that preventable deaths maintained through childhood vaccination programs exceed the risk of death due to COVID-19 in children. This study suggested that although other factors such as logistics and limitations in staffing and resources, routine immunizations in children should be sustained during the pandemic [13].

With approximately 1.15 million cases (as of February 6, 2020), Indonesia has the highest COVID-19 caseload in South East Asia [14]. Although childhood vaccination coverage in 2019 increased compared to 2018 in the country, researchers expected a reduction in uptake in 2020 due to school closures and parental hesitancy [15]. In May 2020, UNICEF noted a 35\% decrease of DPT3 and measles and rubella (MR1) vaccination nationwide compared to the previous year [16]. Low vaccination coverage, poor awareness of immunization advantages and limited access to vaccination clinics lead to a diphtheria outbreak in 2017 in Indonesia which is why delaying immunization was not recommended [17]. Vaccine hesitancy and acceptance in Indonesia are concerning and need to be addressed by local and national governments, especially during the COVID-19 pandemic [18]. This study aims to assess the extent of childhood vaccination disruptions in a representative sample in Indonesia, as well as to determine the predictors associated with delaying pediatric immunizations during the COVID-19 pandemic.

\section{Methods}

\section{Study design and the survey}

In this cross-sectional study, participants who were 18 years or older and living in Indonesia, were sampled from an online survey research firm, Dynata, between August and December, 2020. This is a closed survey only open to Dynata's sampling frame that was used to recruit participants through social media and direct email messages. Gender and age sampling quotas were set to diversify the samples. The method and result of this study is reported according to the Checklist for Reporting Results of Internet E-Surveys (CHERRIES) [19]. Participating in the study was completely voluntary and participants did not receive any compensation from the researchers but were entitled to receive points and prizes as outlined in participation in the Dynata panel. The calculation of sample size was based on computing descriptive statistics with a margin of error of $4 \%$, with a power of $80 \%$ and an alpha of 0.05 .

\section{Questionnaire}

We developed a bilingual (English and Indonesian) questionnaire to assess parental delays in pediatric immunization during the COVID-19 pandemic, as well as to collect demographic and explanatory variables that might associated with delaying immunization. The questionnaire was adjusted according to the pre-test responses. The final questionnaire took about 10-15 minutes to complete. The questionnaire is available in Supplementary information 1. Datasets were uploaded 
to the Inter-university Consortium for Political and Social Research (ICPSR) database and are available at https://doi.org/10.3886/E130422V1.

\section{Study variables}

The primary response variable of the study was parental delay of childhood immunization during the pandemic. It was measured by asking participating parents whether they ever delayed immunization in their children during the COVID-19 pandemic and for what type of immunization.

There were several groups of explanatory variables assessed in this study including demographic characteristics, current underlying disease of the parents, level of exposure to and confidence in COVID-19 information sources, perceived risk of COVID-19 infection, attitudes and practice on vaccination, and COVID-19 prevention measures. Sociodemographic characteristics included age, gender, religion, urbanicity, parental employment status, healthcare worker status, and household income. Ages of the participants were divided into 18-20, 21-30, 31-40, 41-50, and more than 50 years of age for statistical analysis purposes. Gender was classified into male, female, and other (which was self-defined by the participant). Based on the urbanicity, the participant living condition was divided into urban and rural area. Employment status was divided into employed for wages, self-employed, out of work for more or less than 1 year, homemaker, student, retired, or unable to work. The participant was also asked whether they are a healthcare worker such as a nurse, medical doctor, or pharmacist. The annual household income was categorized into five groups: less than US\$ 500, US\$ 1,000-2,999, US\$ 3,000- 7,999, US\$ 8,000-12,999, and >US\$13,00o. The participant was also asked whether they have any COVID19 comorbidities (self-defined by the respondents), measured by asking the respondent whether they have any chronic illnesses.

To measure exposure to COVID-19 information, the respondents were asked whether they knew people in immediate social environment who are, or have been infected, with COVID-19, and have seen or read about individuals infected with COVID-19 on certain news media, such as television. We also asked how confident the parents felt regarding theirs and the scientific community's current knowledge about COVID-19. Finally, participants were asked about their perceived risk of getting COVID-19 infection in the next month, as well as dying from a COVID19 infection. These final two items were measured on a continuous scale of $0 \%$ to $100 \%$. For statistical analyses, we dichotomized levels of perceived risk to $\leq 50$ and $>50$.

We assessed attitudes towards childhood vaccinations by asking respondents to express their agreement (strongly disagree, disagree, neither agree or disagree, agree and strongly agree) on eight statement questions regarding general knowledge on vaccination and whether they follow their doctor's recommendations. For each, the most positive attitude answer was scored 5 while the most negative attitude was scored 1 ; therefore, the total scores ranged between 8 to 40 . Those who achieved more than $80 \%$ out of 40 score was classified had a good attitude towards vaccination; those who answered otherwise were classified as having poor attitudes.

To measure practice of COVID-19 prevention behaviors during the pandemic, respondents were asked how often they went out to work or school outside of the home, or to a grocery store or other food vendors during a typical week. For each activity, the possible answers ranged from o to 7 times a week (i.e., the total scores were o to 14 times a week). Those who went out less than 4 times for both those activities a week were classified had good COVID-19 prevention practices; those who answered otherwise were classified as having poor COVID-19 prevention practices. The parents were also asked whether they have ever been diagnosed as COVID-19 by a doctor.

\section{Statistical analysis}

To determine the possible variables associated with immunization delaying in Indonesian parent during the pandemic, logistic regression was employed. The analysis was conducted in two steps. In the initial step, all independent variables were included in the univariate logistic regression model. In the next step, all variables with $\mathrm{p}<0.05$ on univariate model were then included in multivariate logistic regression. Odd ratios (OR) and 95\% confidence intervals (95\% CI) was calculated. All analyses were conducted in using SPSS version 20 (IBM, New York, USA). 


\section{Results}

\section{Respondents characteristics}

We received 1565 responses during the time period, of which 428 them were excluded due to incomplete data, yielding a final sample size of 1137 for analysis. The socio-demographic characteristics of respondents are presented in Table 1. A third (30.3\%) of respondents were between $31-40$ years old and $28.1 \%$ were between $41-50$ years old. There is a slightly equal number between males and females in this study (52.6 vs. 46.2\%). The majority of respondents were Muslim (78.2\%) and living in an urban area (75.5\%). Almost half of the respondents were self-employed (47.4\%) and only 3.4\% of all respondents were healthcare workers. More than onethird of respondents earned less than $\$ 3,000$ a month. There were 45 (4\%) participants who selfdefined as having a chronic illness.

Although the vast majority (more than $85 \%$ ) of participants have seen or read about individuals infected with COVID-19 on social media, TV, or newspaper, only $22.1 \%$ of the participant felt very confident that they were well-informed about COVID-19. The perceived risk of contracting or dying from COVID-19 was still low (19.9\% and $15.6 \%$, respectively), and further reflected in nearly $70 \%$ of participants that poorly practiced preventative measures against COVID-19. Despite $57.2 \%$ of participants felt somewhat confident that scientists were well informed about COVID-19, only 31.1\% of the participants practiced good COVID-19 prevention measures as suggested by the experts. Almost a third (32.2\%) of respondents knew of individuals infected with COVID-19 among their immediate social environment, moreover, $29.7 \%$ of all participants tested positive for COVID-19. About $60 \%$ of all participants expressed poor attitudes towards vaccination which reflects how only $32.6 \%$ of all participants received a flu shot during the previous season.

\section{Magnitude of childhood vaccination disruption practices in Indonesia}

About $42 \%$ of respondents stated that they ever heard that the local health facilities in their area stopped childhood vaccination service for kids due to the pandemic. Approximately $13.3 \%$ (193/1137) respondents stated that their children could not be vaccinated because healthcare facilities temporarily stopped vaccination services during the pandemic. Out of total respondents, 312 of them (27.4\%) delayed bringing their children to get compulsory vaccinations from local healthcare facilities during the COVID-19 pandemic.

\section{Factors associated with delaying childhood vaccination during the COVID-19} pandemic in Indonesia

In unadjusted analyses, women were 1.36 times more likely to delay vaccination for their children compared to men (95\%CI: 1.04, 1.76) (Table 1). Homemakers, retirees, or those unable to work had lower odds of delaying compulsory immunization for their children compared to those employed for wages, OR: 0.60, 95\%CI: 0.37, 0.98 and OR: 0.13; 95\%CI: 0.04, 0.43, respectively. Compared to participants who were working in non-healthcare sectors, healthcare workers were 4.02 times more likely to delay their children's vaccination (95\%CI: 2.10, 7.72). Parents who reported having a household income of $\$ 1,000-\$ 2,999$ and $\$ 3,000-\$ 7,999$ monthly had higher odds of postponing their children's immunizations during the pandemic by 1.54 and 1.73 times, respectively, compared to those who earned less than $<\$ 500$ a month. Compared to those who had no comorbidity, participants who had chronic disease or those who were unsure of having any comorbidity had higher odds of delaying childhood vaccination, 2.7 and 2.0 times, respectively.

Exposure to COVID-19 information is also associated with delaying childhood vaccination. For example, if the respondents know people in immediate social environment who are or have been infected with the COVID-19, they were nearly 1.5 times more likely to delay their children's vaccination. In addition, those with exposure to COVID-19 information from social media, TVnewspapers also had higher odds of delaying childhood immunization compared to their counterparts who had no exposure (OR: 1.68, 95\%CI: 1.01, 2.78 and OR: 2.12, 95\%CI: 1.33, 3.38, respectively). 
Table 1. Unadjusted and multivariable logistic regression analysis showing predictors of parents delaying immunization of their children during COVID-19 pandemic $(n=1137)$

\begin{tabular}{|c|c|c|c|c|c|c|}
\hline \multirow[t]{2}{*}{ Variable } & \multirow[t]{2}{*}{$\mathrm{n}(\%)$} & \multirow{2}{*}{$\begin{array}{l}\text { Delaying immunization } \\
\mathrm{n}(\%)\end{array}$} & \multicolumn{2}{|l|}{ Unadjusted } & \multicolumn{2}{|l|}{ Multivariable } \\
\hline & & & OR $(95 \% \mathrm{CI})$ & $\mathrm{p}$-value & $\mathrm{OR}(95 \% \mathrm{CI})$ & $\mathrm{p}$-value \\
\hline \multicolumn{7}{|l|}{ Age group (vear) } \\
\hline $18-20$ (Reference group $(R)$ ) & $52(4.6)$ & $15(28.8)$ & 1 & & 1 & \\
\hline $21-30$ & $268(23.6)$ & $99(36.9)$ & $1.45(0.76,2.77)$ & 0.266 & $1.32(0.61,2.84)$ & 0.479 \\
\hline 31,40 & $345(30.3)$ & $118(34.2)$ & $1.28(0.68,2.43)$ & 0.446 & $1.39(0.64,3.02)$ & 0.410 \\
\hline 41,50 & $319(28.1)$ & $58(18.2)$ & $0.55(0.28,1.07)$ & 0.076 & $0.64(0.29,1.43)$ & 0.276 \\
\hline$>50$ & $153(13.5)$ & $22(14.4)$ & $0.41(0.20,0.88)$ & $0.021^{*}$ & $0.55(0.23,1.32)$ & 0.178 \\
\hline \multicolumn{7}{|l|}{ Gender } \\
\hline Male $(R)$ & $598(52.6)$ & $148(24.7)$ & 1 & & 1 & \\
\hline Female & $525(46.2)$ & $162(30.9)$ & $1.36(1.04,1.76)$ & $0.023^{*}$ & $1.27(0.94,1.71)$ & 0.120 \\
\hline \multirow{2}{*}{\multicolumn{7}{|c|}{ Religion }} \\
\hline & & & & & & \\
\hline Islam $(R)$ & $889(78.2)$ & $259(29.1)$ & 1 & & & \\
\hline Protestant & $71(6.2)$ & $14(19.7)$ & $0.60(0.33,1.09)$ & 0.094 & & \\
\hline $\begin{array}{l}\text { Christian } \\
\text { Cla }\end{array}$ & $148(13.0)$ & $34(23.0)$ & $0.73(0.48,1.09)$ & 0.124 & & \\
\hline Others (including Hinduism, Buddhism and Khongsuhu) & $29(2.6)$ & $5(17.2)$ & $0.51(0.19,1.34)$ & 0.172 & & \\
\hline \multicolumn{7}{|l|}{ Location of living } \\
\hline $\operatorname{Urban}(R)$ & $858(75.5)$ & $232(27.0)$ & 1 & & & \\
\hline Rural (in the country) & $279(24.5)$ & $80(28.7)$ & $1.09(0.80,1.46)$ & 0.595 & & \\
\hline \multicolumn{7}{|l|}{ Employment } \\
\hline Employed for wages $(R)$ & $334(29.4)$ & $105(31.4)$ & 1 & & 1 & \\
\hline Self, employed & $539(47.4)$ & $155(28.8)$ & $0.88(0.65,1.19)$ & 0.400 & $0.78(0.56,1.07)$ & 0.126 \\
\hline Out of work for both less or more than 1 year & $50(4.4)$ & $11(22.0)$ & $0.62(0.30,1.25)$ & 0.179 & $0.60(0.28,1.29)$ & 0.193 \\
\hline Homemaker & $125(11.0)$ & $27(21.6)$ & $0.60(0.37,0.98)$ & $0.039^{* *}$ & $0.57(0.33,0.98)$ & $0.043^{*}$ \\
\hline Student & $36(3.2)$ & $11(30.6)$ & $0.96(0.46,2.02)$ & 0.914 & $0.72(0.30,1.74)$ & 0.464 \\
\hline Retired or unable to work & $53(4.7)$ & $3(5.7)$ & $0.13(0.04,0.43)$ & $0.001^{* * *}$ & $0.21(0.06,0.72)$ & $0.013^{*}$ \\
\hline \multicolumn{7}{|l|}{ Healthcare worker (nurse, medical doctor or pharmacist) } \\
\hline No $(R)$ & $1098(96.6)$ & $289(26.3)$ & 1 & & 1 & \\
\hline Yes & $39(3.4)$ & $23(59.0)$ & $4.02(2.10,7.72)$ & $<0.001^{* * *}$ & $1.82(0.87,3.81)$ & 0.114 \\
\hline \multicolumn{7}{|l|}{ Household income last year from all sources before taxes } \\
\hline Less than $\$ 500$ a month $(R)$ & $322(28.3)$ & $70(21.7)$ & 1 & & 1 & \\
\hline$\$ 1,000, \$ 2,999$ a month & $437(38.4)$ & $131(30.0)$ & $1.54(1.10,2.15)$ & $0.011^{*}$ & $1.40(0.97,2.02)$ & 0.069 \\
\hline$\$ 3,000, \$ 7,999$ a month & $200(17.6)$ & $65(32.5)$ & $1.73(1.17,2.58)$ & $0.007^{* *}$ & $1.51(0.97,2.35)$ & 0.068 \\
\hline$\$ 8,000, \$ 12,999$ a month & 125 (11.0) & $33(26.4)$ & $1.29(0.80,2.08)$ & 0.294 & $1.09(0.65,1.84)$ & 0.735 \\
\hline$\$ 13,000$ a month or more & $53(4.7)$ & $13(24.5)$ & $1.17(0.59,2.31)$ & 0.651 & $1.02(0.49,2.12)$ & 0.965 \\
\hline \multicolumn{7}{|l|}{ Have a chronic illness } \\
\hline No $(R)$ & $1054(92.7)$ & $274(26.0)$ & 1 & & 1 & \\
\hline Yes & $45(4.0)$ & $22(48.9)$ & $2.72(1.49,4.96)$ & $0.001^{* * *}$ & $2.03(0.99,4.15)$ & 0.054 \\
\hline $\begin{array}{l}\text { Do not know } \\
\text { Knowing people in immediate social environment who are or have been } \\
\text { infected with the COVID, } 19\end{array}$ & $38(3.3)$ & $16(42.1)$ & $2.07(1.07,4.00)$ & $0.030^{*}$ & $2.29(1.11,4.71)$ & $0.025^{*}$ \\
\hline
\end{tabular}




\begin{tabular}{|c|c|c|c|c|c|c|}
\hline \multirow[t]{2}{*}{ Variable } & \multirow[t]{2}{*}{ n (\%) } & \multirow{2}{*}{$\begin{array}{l}\text { Delaying immunization } \\
\mathrm{n}(\%)\end{array}$} & \multicolumn{2}{|l|}{ Unadjusted } & \multicolumn{2}{|l|}{ Multivariable } \\
\hline & & & OR (95\% CI) & p-value & OR (95\% CI) & p-value \\
\hline Yes, confirmed $(R)$ & $366(32.2)$ & $129(35.2)$ & 1 & & 1 & \\
\hline No & $642(56.5)$ & $149(23.2)$ & $0.56(0.42,0.74)$ & $<0.001^{* *}$ & $1.08(0.64,1.81)$ & 0.775 \\
\hline Do not know & $129(11.3)$ & $34(26.4)$ & $0.66(0.42,1.03)$ & 0.066 & $0.61(0.29,1.31)$ & 0.206 \\
\hline No $(R)$ & $105(9.2)$ & $20(19.0)$ & 1 & & 1 & \\
\hline Yes & $1032(90.8)$ & $292(28.3)$ & $1.68(1.01,2.78)$ & $0.045^{*}$ & $1.23(0.70,2.16)$ & 0.478 \\
\hline \multicolumn{7}{|c|}{$\begin{array}{l}\text { Have seen or read about individuals infected with COVID-19 on TV or } \\
\text { newspaper }\end{array}$} \\
\hline No $(R)$ & $142(12.5)$ & $23(16.2)$ & 1 & & 1 & \\
\hline Yes & $995(87.5)$ & $289(29.0)$ & $2.12(1.33,3.38)$ & $0.002^{* * *}$ & $1.33(0.79,2.25)$ & 0.280 \\
\hline \multicolumn{7}{|c|}{ Have been infected with the COVID-19 } \\
\hline Yes, confirmed $(R)$ & $338(29.7)$ & $125(37.0)$ & 1 & & 1 & \\
\hline No & $656(57.7)$ & $143(21.8)$ & $0.48(0.36,0.63)$ & $<0.001^{* *}$ & $0.55(0.32,0.93)$ & $0.026^{*}$ \\
\hline Do not know & $143(12.6)$ & $44(30.8)$ & $0.76(0.50,1.15)$ & 0.193 & $1.21(0.59,2.48)$ & 0.605 \\
\hline \multicolumn{7}{|c|}{ Confidence in how much knowing about COVID-19 } \\
\hline Very confident $(R)$ & $251(22.1)$ & $73(29.1)$ & 1 & & 1 & \\
\hline Not confident & $136(12.0)$ & $26(19.1)$ & $0.58(0.35,0.96)$ & $0.033^{*}$ & $0.82(0.47,1.44)$ & 0.490 \\
\hline Somewhat confident & $750(66.0)$ & $213(28.4)$ & $0.97(0.71,1.33)$ & 0.836 & $1.35(0.95,1.91)$ & 0.097 \\
\hline \multicolumn{7}{|c|}{ Confidence in how much scientists and experts know about COVID-19 } \\
\hline Somewhat confident & $650(57.2)$ & $173(26.6)$ & $0.82(0.62,1.09)$ & 0.176 & & \\
\hline \multicolumn{7}{|c|}{ Score of perceived risk of getting COVID-19 infection in the next month } \\
\hline$\leq 50(R)$ & $911(80.1)$ & $233(25.6)$ & 1 & & 1 & \\
\hline$>50(R)$ & $226(19.9)$ & $79(35.0)$ & $1.56(1.15,2.14)$ & $0.005^{* *}$ & $1.20(0.83,1.75)$ & 0.327 \\
\hline \multicolumn{7}{|c|}{ Score of perceived risk of dying from COVID-19 if infected } \\
\hline $0-50(R)$ & $960(84.4)$ & $250(26.0)$ & 1 & & 1 & \\
\hline$>50$ & $177(15.6)$ & $62(35.0)$ & $1.53(1.09,2.15)$ & $0.014^{*}$ & $1.02(0.67,1.55)$ & 0.932 \\
\hline \multicolumn{7}{|c|}{ Have received a flu shot during the past 12 months } \\
\hline Yes $(R)$ & $371(32.6)$ & $136(36.7)$ & 1 & & 1 & \\
\hline No & $766(67.4)$ & $176(23.0)$ & $0.52(0.39,0.68)$ & $<0.001^{* *}$ & $0.70(0.51,0.95)$ & $0.021^{*}$ \\
\hline \multicolumn{7}{|c|}{ Attitude level to vaccination } \\
\hline $\operatorname{Good}(R)$ & $461(40.5)$ & $140(30.4)$ & 1 & & & \\
\hline Poor & $676(59.5)$ & $172(25.4)$ & $0.78(0.60,1.02)$ & 0.068 & & \\
\hline \multicolumn{7}{|c|}{ Practice level on COVID, 19 prevention } \\
\hline Good $(R)$ & $354(31.1)$ & $91(25.7)$ & 1 & & & \\
\hline Poor & $783(68.9)$ & $221(28.2)$ & $1.14(0.86,1.51)$ & 0.378 & & \\
\hline
\end{tabular}

CI: confidence interval, OR: odds ratio, R: reference group

* Significant at 0.05

** Significant at 0.01 
Those with higher perceived risk of either getting COVID-19 infection in the next month or dying from COVID-19 had higher odds of delaying vaccination compared to those with low perceived risk (OR: 1.56, 95\%CI: 1.15, 2.14 and OR: 1.53, 95\%CI: 1.09, 2.15, respectively). Additionally, respondents who received a flu shot during the previous flu season were more likely to delay compulsory childhood vaccinations during the pandemic (OR: 1.59; 95\%CI: 1.24, 2.06). Participants that have been diagnosed with confirmed COVID-19 were 1.7 times more likely to delay their children's vaccinations compared to those who had never been diagnosed (Table 1).

In multivariate analyses, our data suggest that type of job, presence of chronic disease (i.e., comorbidities for COVID-19), previous flu vaccination, and experience with a COVID-19 diagnosis were associated with delayed childhood vaccination among respondents. Homemakers (aOR: 0.57, 95\%CI: 0.33, 0.98) and retirees (aOR: 0.21, 95\%CI: 0.06, 0.72) demonstrated better compliance with childhood vaccination schedules during the pandemic, compared to those employed for wages. Those unsure of whether they had a chronic illness were 2.29 times more likely to postpone vaccinating their children (aOR: 2.29 , 95\%CI: 1.11, 4.71). Respondents who received a flu shot during the previous season were also more likely to delay vaccinating their children during the pandemic. Participants diagnosed COVID-19 were more likely to delay their children's vaccinations compared to those who had never been diagnosed.

\section{Discussion}

Vaccination during the pandemic is a concerning issue in Indonesia, not only due to mitigation strategies that limit in-person interactions, but also due to the financial instability of immunization programs during the pandemic [15]. We conducted this nationwide, crosssectional study to assess the magnitude of routine childhood vaccination disruption and to determine possible factors influencing the delay of compulsory vaccination in children during the COVID-19 pandemic. Our results find that homemakers and retirees had significantly better compliance with their children's vaccination schedules during the pandemic compared to those employed for wages. This finding is similar to another study concluding that housewives approved of vaccines more so compared to farmers [18]. In contrast, another study reported that unemployment was not a significant predictor of parents' attitudes towards routine vaccination during the emergence of Zika virus in Indonesia [20].

Delaying childhood vaccination was 2.3 times higher among those unsure of whether they had COVID-19 comorbidity. Hesitancy among this group might be related to evidence that COVID-19 mortality is higher in people with underlying medical comorbidities like diabetes, cancer, hypertension, heart, lung, and kidney diseases [21-24]. The odds of disruption of childhood immunization schedules were higher among individuals who are or have been infected with COVID-19 or had immediate social contacts who are or have been infected with COVID-19 than those who have not been infected. Difficulty in attending vaccination appointments might be influenced by parents who develop COVID-19 symptoms and consequently self-isolate for 2 weeks, or who are caring for a member of their household infected with COVID-19 [25].

Risk perceptions can greatly influence preventive behaviors [26, 27]. Our univariate analyses show that people who perceived a higher risk in contracting or dying from COVID-19 delayed their children's routine vaccination. This might be attributed to national recommendations to stay at home as a preventative measure against COVID-19. One study reported similar findings; the stay-at-home orders and social distancing measures implemented to stop the spread of COVID-19 reduced immunization activities, resulting in decreased pediatric vaccination uptake [28]. A study in Michigan, USA, reported that vaccination coverage among children $\leq 2$ years old declined below 50\% after the state implemented social distancing and quarantine policies [4]. Parents may be postponing childhood vaccinations based on perceived risk of contracting SARSCoV-2 in a public place. Given an inattention to other countermeasures - evident by $68.9 \%$ of study participants practicing inadequate preventative measures, such as not wearing masks or observing physical distancing - these concerns may be warranted. Moreover, it could be that parents are concerned about their children's vulnerability to COVID-19 [29]. However, overall, it is important for childhood vaccination services to remain running during a pandemic like COVID19 , where the burden of disease is relatively low in children. 
This study also found that participants who got a flu shot in the previous season were more likely to delay their children's vaccination. This finding is different from previous studies that showed that parents with better attitudes towards vaccination were more likely to have their children vaccinated [30-33]. At the beginning of the pandemic, demand of flu vaccination increased in Indonesia since both influenza and COVID-19 share similar signs and symptoms [34] and some people thought that the flu shot could protect them from the new disease. A previous study also suggested that the COVID-19 pandemic increased willingness to receive the influenza vaccine [34]. Individuals who believed they were more likely to be infected with COVID-19 had increased uptake of influenza vaccine in both children and their caregivers [35]. On the contrary, parents who got the flu shot were more likely to have higher risk-perception of COVID-19, thus they could be more likely to avoid visiting health centers out of fear of catching the virus. This could be one of the reasons behind our study's finding.

Another possible explanation for the disruption of routine vaccinations among Indonesian parents is may be due to the high volume of reporting on COVID-19, a novel and unfamiliar disease, on the news or social media [36]. This might result in heightened anxiety and misperception of COVID-19 as more severe, which should then be avoided at all cost, in this case, by minimizing contacts with others, including vaccination officers [37]. We observed this in our study, which showed higher odds of disruption of childhood immunizations in those with heightened exposure to COVID-19 updates from social media or news, by 1.68 and 2.12, respectively. Moreover, insufficient information about the consequences of declining pediatric vaccine coverage during this pandemic might lead to the reversal of years of achievement in eliminating vaccine-preventable disease and death [38]. Based on the UNICEF data, the underfive mortality rate in Indonesia has been declined from $52.10 \%$ in 2010 to $50.80 \%$ in 2018 [39]. Twenty-one countries in East Asia and Pacific reported disruptions of health services during the pandemic with Palau and Myanmar experienced the highest drop on routine vaccination by 50$74 \%$ and $25-49 \%$, respectively [40]; however, no data on how routine vaccination change during the pandemic in Indonesia.

This study has a number of limitations. First, the data are derived from an internet-based, conveniently sampled survey. Despite the presence of sampling quotas particularly for age and gender identity, we removed any participants who did not consent, did not complete most of the survey, and those who rapidly took the survey. While we implemented best practices to derive a functional and valid dataset, the loss of participants from the original raw set impacts the representability of the sample. Verification of our results should be confirmed with repeated measures, population-based samples, and larger sample sizes. Additionally, because the data used are cross sectional, measuring temporality in our research question is improbable, thus the question of causality remains. To assess dynamic changes in parental attitudes towards childhood vaccinations during the COVID-19 pandemic, longitudinal data that depict the dynamics of vaccine decision-making in parents are required.

That said, this study is part of a growing body of research illustrating the impact of global upheavals such as a pandemic on childhood vaccination schedules [12]. To our knowledge this study is the first of its kind to examine the status and determinants of vaccine decision-making in parents during the COVID-19 pandemic in Indonesia.

Health literacy, trust in health authority recommendations, and health messaging influence individual risk perception and vaccination decision-making [41]. Therefore, campaigns regarding vaccine availability, accessibility, and efficacy should be well presented to the community in order to minimize the information gap which risks outbreaks of vaccine-preventable diseases in Indonesia [15]. This phenomenon was also seen in the UK, where people felt that more information about preventative measures taken by staff to ensure public safety while attending vaccination appointments during the pandemic should be communicated [25]. A previous study has also explained further strategies to bolster a community's belief in the relevance and importance of immunization during a pandemic [16]. 


\section{Conclusion}

Indonesia experienced a significant disruption of routine childhood immunizations, which was not only attributed to the limited health services but also due to parental decisions to delay vaccinating their children. Vaccination coverage should immediately recover through implementing better strategies, not only by increasing vaccination services, but also through improving the parents' perceptions and confidence towards childhood immunizations during the pandemic.

\section{Declarations}

\section{Ethics approval}

Ethical approval was granted by Institutional Review Board of Universitas Syiah Kuala, (041/EA/FK-RSUDZA/2020) and the National Health Research and Development Ethics Commission of the Ministry of Health of the Republic of Indonesia (\#1171012P).

\section{Acknowledgments}

We would like to thank to all participants who took part in this survey.

\section{Conflict of interest}

The authors declare that they have no competing interests.

\section{Funding}

This project was supported by an award from the National Science Foundation, Division of Social and Economic Sciences (\#2027836).

\section{How to cite}

Fahriani M, Anwar S, Yufika A, Bakhtiar B, et al. Disruption of childhood vaccination during the COVID-19 pandemic in Indonesia. Narra J 2021; 1 (1): e7. http://doi.org/10.52225/narraj.v1i1.7.

\section{References}

1. WHO. Immunization Agenda 2030: A global strategy to leave no one behind. 2019. Available from: https://www.who.int/publications/m/item/immunization-agenda-2030-a-global-strategy-to-leave-no-one-behind (Accessed: 1 January 2021)

2. Harapan H, Shields N, Kachoria AG, et al. Religion and measles vaccination in Indonesia, 1991-2017. Am J Prev Med 2021; 60(1 Suppl 1):S44-S52.

3. WHO. WHO Director-General's opening remarks at the media briefing on COVID-19-11 March 2020. 2020. Available from: https://www.who.int/director-general/speeches/detail/who-director-general-s-opening-remarks-at-themedia-briefing-on-covid-19---11-march-2020 (Accessed: 1 January 2021).

4. Bramer CA, Kimmins LM, Swanson R, et al. Decline in child vaccination coverage during the COVID-19 pandemic Michigan Care Improvement Registry, May 2016-May 2020. 2020; 68(22)

5. Dabo M, Samah Y, Kande M, et al. Early effect of the Covid-19 epidemic on vaccine coverage of major antigens in Guinea: an analysis of the interrupted time series of national immunization coverage. medRxiv 2020. https://doi.org/10.1101/2020.09.11.20192161.

6. McDonald HI, Tessier E, White JM, et al. Early impact of the coronavirus disease (COVID-19) pandemic and physical distancing measures on routine childhood vaccinations in England, January to April 2020. Eurosurveillance 2020; 25(19):2000848.

7. WHO. WHO and UNICEF warn of a decline in vaccinations during COVID-19. 2020. Available from: https://www.who.int/news/item/15-07-2020-who-and-unicef-warn-of-a-decline-in-vaccinations-during-covid-19 (Accessed: 1 January 2021). 
8. Alrabiaah AA, Alshaer AH, Estrella SMC, et al. Effects of the coronavirus disease 2019 pandemic on routine pediatric immunization coverage rates at the main University Hospital in Saudi Arabia. Saudi Med J 2020; 41:1197.

9. Ali I. Impact of COVID-19 on vaccination programs: adverse or positive? Human Vaccines \& Immunotherapeutics 2020; 16(11):2594-2600.

10. Saxena S, Skirrow H, Bedford H. Routine vaccination during covid-19 pandemic response. BMJ 2020; 369:m2392.

11. Santoli JM. Effects of the COVID-19 pandemic on routine pediatric vaccine ordering and administration-United States, 2020. MMWR Morb Mortal Wkly Rep 2020;69(19):591-593

12. Chandir S, Siddiqi DA, Mehmood M, et al. Impact of COVID-19 pandemic response on uptake of routine immunizations in Sindh, Pakistan: An analysis of provincial electronic immunization registry data. Vaccine 2020; 38(45):7146-7155.

13. Abbas K, Procter SR, van Zandvoort K, et al. Routine childhood immunisation during the COVID-19 pandemic in Africa: a benefit\&\#x2013;risk analysis of health benefits versus excess risk of SARS-CoV-2 infection. Lancet Global Health 2020; 8(10):e1264-e1272.

14. Kemenkes. The current update on COVID-19 cases. Availabe from: https://www.kemkes.go.id/index.php?lg=LN02 (Accessed: 1 January 2021).

15. Suwantika AA, Boersma C, Postma MJ. The potential impact of COVID-19 pandemic on the immunization performance in Indonesia. Expert Review of Vaccines 2020; 19(8):687-690.

16. The Ministry of Health and UNICEF Indonesia. Routine immunization for children during the COVID-19 pandemic in Indonesia: perceptions of parents and caregivers. 2020. Available from: https://www.unicef.org/indonesia/reports/routine-immunization-children-during-covid-19-pandemic-indonesia (Accessed: 1 January 2021).

17. Harapan H, Anwar S, Dimiati H, et al. Diphtheria outbreak in Indonesia, 2017: An outbreak of an ancient and vaccinepreventable disease in the third millennium. Clinical Epidemiol Glob Health 2019; 7(2):261-262.

18. Harapan H, Alleta A, Anwar S, et al. Socio-demographic factors associated with inhabitants' view related to vaccines in Indonesia. Iranian journal of public health 2017; 46(5):708-710.

19. Eysenbach G. Improving the Quality of Web Surveys: The Checklist for Reporting Results of Internet E-Surveys (CHERRIES). J Med Internet Res 2004; 6(3):e34.

20. Yufika A, Wagner AL, Nawawi Y, et al. Parents' hesitancy towards vaccination in Indonesia: A cross-sectional study in Indonesia. Vaccine 2020; 38(11):2592-2599.

21. Murthy S, Gomersall CD, Fowler RA. Care for Critically III Patients With COVID-19. JAMA 2020; 323(15):1499-1500.

22. Zhou F, Yu T, Du R, et al. Clinical course and risk factors for mortality of adult inpatients with COVID-19 in Wuhan, China: a retrospective cohort study. The Lancet 2020; 395(10229):1054-1062.

23. Wu C, Chen X, Cai Y, et al. Risk Factors Associated With Acute Respiratory Distress Syndrome and Death in Patients With Coronavirus Disease 2019 Pneumonia in Wuhan, China. JAMA Internal Medicine 2020; 180(7):934-943.

24. Yang J, Zheng Y, Gou X, et al. Prevalence of comorbidities and its effects in patients infected with SARS-CoV-2: a systematic review and meta-analysis. International Journal of Infectious Diseases 2020; 94:91-95.

25. Bell S, Clarke R, Paterson P, et al. Parents' and guardians' views and experiences of accessing routine childhood vaccinations during the coronavirus (COVID-19) pandemic: A mixed methods study in England. PLOS ONE 2021; 15(12):e0244049.

26. Fischhoff B. Risk analysis and human behavior: Routledge; 2013.

27. Moen B-E. Explaining risk perception. An evaluation of the psychometric paradigm in risk perception research. Rotunde Publikasjoner, Norway; 2004.

28. Olorunsaiye CZ, Yusuf KK, Reinhart K, et al. COVID-19 and child vaccination: A systematic approach to cosing the immunization gap. Int J MCH AIDS 2020; 9(3):381-385.

29. Bond L, Nolan T. Making sense of perceptions of risk of diseases and vaccinations: a qualitative study combining models of health beliefs, decision-making and risk perception. BMC Public Health 2011; 11(1):943.

30. Matta P, Mouallem RE, Akel M, et al. Parents' knowledge, attitude and practice towards children' s vaccination in Lebanon: role of the parent-physician communication. BMC Public Health 2020; 20:1439.

31. Tobin-West $\mathrm{Cl}$, Alex-Hart BA. Identifying barriers and sustainable solution to childhood immunization in Khana local government area of Rivers State, Nigeria. Int Q Community Health Educ 2011; 32(2):149-158.

32. Smith PJ, Chu SY, Barker LE. Children who have received no vaccines: who are they and where do they live? Pediatrics 2004; 114(1):187-195. 
Fahriani et al. Narra J 2021; 1 (1): e7 - http://doi.org/10.52225/narraj.vii1.7

33. Herath NC, Kudagammana T, Sanathchandra TT, et al. Brief report: parental attitudes and knowledge on routine childhood immunization: an experience from Central Sri Lanka. BMC Res Notes 2018; 11(1):402.

34. Domnich A, Cambiaggi M, Vasco A, et al. Attitudes and beliefs on influenza vaccination during the COVID-19 pandemic: results from a representative Italian survey. Vaccines (Basel) 2020; 8(4):711.

35. Goldman RD, McGregor S, Marneni SR, et al. Willingness to vaccinate children against influenza after the coronavirus disease 2019. Pandemic. J Pediatr 2021;228:87-93.

36. Asmundson GJG, Taylor S. Coronaphobia: Fear and the 2019-nCoV outbreak. J. Anxiety Disord 2020; 70:102196.

37. Paek H-J, Hove T. Risk perceptions and risk characteristics. In: Oxford Research Encyclopedia of Communication. 2017.

38. UNICEF. COVID-19 could reverse decades of progress toward eliminating preventable child deaths, agencies warn. 2020. Available from https://www.who.int/news/item/09-09-2020-covid-19-could-reverse-decades-of-progresstoward-eliminating-preventable-child-deaths-agencies-warn (Accessed 1 March 2021).

39. UNICEF. Child mortality data. 2020. Available from: https://data.unicef.org/topic/child-survival/under-five-mortality/. (Accessed: 1 January 2021).

40. UNICEF. Tracking the situation of children during COVID-19. 2020. Available from: https://data.unicef.org/resources/rapid-situation-tracking-covid-19-socioeconomic-impacts-data-viz/ (Accessed: 1 January 2021).

41. WHO. Vaccination and trust-How concerns arise and the role of communication in mitigating crises. 2017. Available from: https://www.euro.who.int/_data/assets/pdf_file/0004/329647/Vaccines-and-trust.PDF (Accessed: 1 January 2021). 